\title{
Empirical Study on the Demonstration Project Construction of IOTIPS
}

\author{
XIA Chuan-kun, MEI Lin-chang \\ Hebi Power Supply Company, Electric Power Company of Henan Province, State Grid, Hebi, 458030, China
}

\begin{abstract}
This paper describes the current status of power grid and the necessity of the demonstration project construction of IOTIPS (Internet of Things in Power Systems), and proposes the overall demand for the construction of IOTIPS. Taking Ningxia Electricity and Henan's Hebi Electricity as the examples of demonstration project applying IOTIPS, this paper also summarizes the construction achievement of IOTIPS. KEYWORDS: distribution network lines; IOTIPS (Internet of Things in Power Systems); smart grid
\end{abstract}

\section{INTRODUCTION}

In order to standardize the different proprietary communication protocols applied by different equipment manufacturers in the communication among grid devices, to make the sensing devices and mobile terminals made by all manufacturers be smoothly connected to the grid, unified information model, unified communication protocol, unified data services and application services based on Internet of Things are proposed, which greatly facilitates the communications among devices and the selection of equipment, and promotes the promotion of new technologies and products.

As the experimental places of 2013 demonstration project construction applying IOTIPS, and based on the construction achievement of 2012 demonstration construction applying Internet of Things, Ningxia Electricity and Henan's Hebi Electricity carry out deepening construction on the demonstration projects applying Internet of Things. Based on Internet of Things technology, on-line monitoring, condition-based maintenance and lifecycle management of distribution network equipment are discussed below.

\section{NECESSITY OF THE DEMONSTRATION PROJECT CONSTRUCTION OF IOTIPS}

\subsection{Meeting the Needs of Information Perception and Unified Managing in All Aspects of Power Grid}

Online monitoring of the important operating parameters in all aspects of the grid, the prediction, prevention and control of device state, establishing an auxiliary decision of transmission lines and an intelligent decision of distribution aspects based on reliable monitoring information, strengthening twoway interaction between users, opening up new value-added service and so on are part of the core tasks of smart grid construction. And the achievement of these intelligent tasks must rely on thorough information perception, reliable data transmission, sound network infrastructure, intelligent management of vast amounts of information and efficient processing of multi-level data. Currently, the technologies of Internet of Things like radio frequency identification, wireless sensor networks and global positioning technology are used in all aspects like power system production, management and so on, to help achieve an efficient grid situation awareness, and improve management capabilities of power standardization.

\subsection{Meeting the Needs of Perceptive Technology in the Large-Scale Construction of Smart Grid}

Mutual penetration, deep integration and wide application of smart grid and Internet of Things can effectively integrate communications infrastructure resources and power system infrastructure resources, improve information level, level of safe operation and level of reliable power supply and excellent service of power system, reduce the line loss, and improve power transmission efficiency and utilization efficiency; also can drive the development of intelligent terminals, smart sensors, information and communications equipment, power chips, software as well as operation and maintenance industry, enhance independent innovation capability, market competitiveness and technical support 
capabilities of the industrial units supervised, and make the industries directly under state grid bigger and stronger.

IOTIPS widely arranges various intelligent sensing devices of a certain sensing capability, computational capability and performing capability in all aspects of power production, transmission, consumption and management, uses IP-based standard protocol to achieve safe and reliable transmission, co-processing, unified services and application integration of information through the power information communication networks, so as to realize panoramic holographic perception, interoperability and seamless integration of power grid operation and business management's whole process. [1]

\subsection{Meeting the Needs of Unified Planning and Construction and Unified Framework Design of IOTIPS}

The content involved in Internet of Things is allinclusive, and IOTIPS covers all aspects of the power grid. But so far, there has been no unified development plan of IOTIPS, the overall framework of IOTIPS still need to be improved, and the application mode need to be further clarified. To early arrange and plan the development plan of power information communication, and carry out study relating Internet of Things of power systems, it is particularly important to conduct unified planning of IOTIPS and establish framework. As the outline of IOTIPS development, the planning content will focus on the information communication needs of all aspects of power systems and the management needs of grid enterprises, and emphasize the study on the technology framework of IOTIPS, and the architecture of application system, information communication system and key technologies of IOTIPS. It will be able to effectively integrate communications infrastructure resources and power system infrastructure resources, to make information communication serve in power system operation, improve the information level of power system, and improve the utilization efficiency of existing power system infrastructure. [2]

\subsection{IOTIPS Technology Involves a Large Number of Sophisticated Technology and Scenario Adaptation, is a Huge Systematic and Practical Project, and Needs to Conduct Application Demonstration as a Support.}

Demonstration project construction of Internet of Things is based on the overall framework of IOTIPS, which plays an important demonstration role in the verification of improving smart grid's ability in information collection, intelligent procession of information, and two-way communication of information in the major aspects of power transmission, substation, distribution and consumption. A large number of standards, technical validation and scenarios application demonstration will be developed through the construction of regional application demonstration project, which offers reproducible and operational real experience for the promotion of IOTIPS, and also has a positive effect for the construction of smart grid.

\section{DEMONSTRATION PROJECT DESIGN OF IOTIPS}

\subsection{Overall Architecture of IOTIPS}

The overall architecture of IOTIPS is based on the overall architecture of State Grid "SG-ERP", including perception layer, network layer and application layer. The next main research work of IOTIPS is to achieve the unified information model and unified communications protocol of perception layer and network layer, and to make application layer achieve a unified application services for the application of other business system.

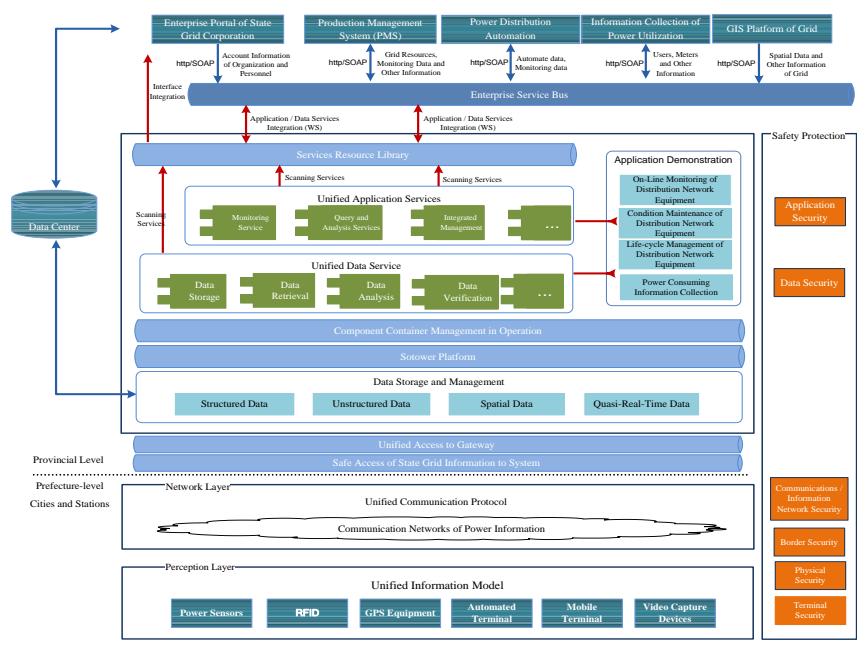

Figure 1 The power of Internet of things the president architecture

Perception layer mainly applies various sensors to achieve information collection, identification and integration. Its research focus is to achieve a unified information model, specifically including unified identity, unified semantics, unified data representation format, terminal security and other aspects, forming the relevant standards, and researching and developing standardized collection terminals, standardized communication modules and information format conversion equipment.

Network layer is responsible for the transmission and bearing of information obtained from perception layer. Network layer aims to introduce a variety of converged communication technologies, studies and develops standardized communication protocols and gateway devices on the basis of wealthy 
communication modes. In the application, sensors and sink node are connected by micro-power wireless communication technologies, to solve problems of information collection coverage and flexibility, and sink node and access gateway are connected through optical networks, PLC, wireless broadband and so on, to solve the problems of longdistance information transmission and reliability.

Based on SG-ERP architecture of State Grid, application layer establishes data centers, researches unified data model of IOTIPS, and achieves the function of releasing a unified data service and packaging system, providing all kinds of unified application services for existing business systems, and also providing a higher level of service for other business systems.[3]

\subsection{On-Line Monitoring of Distribution Network Equipment}

\subsubsection{Construction Content}

It is to achieve operational status and environment monitoring on transformers, RMU, cable branch box, pole isolating switch, pole circuit breakers, segmented lines, buried cables and so on, which can provide support for the services like operation and management, fault warning, fast fault location, etc. of distribution network equipment.

\subsubsection{Communication Scheme}

Sensors collect power distribution equipment's operating environment data and operating status data, and then transmit the data to the nearest shortrange wireless backbone nodes through its own wireless communication module, and through the self-organizing network combined by multiple backbone nodes the data is transmitted to data transmission base. And then through the matching ONU optical communication of power distribution automation system, the information is introduced to the substation controller, and enters electricity security zone III to the master data collection server through data convergence controller in the substation controller.[4]

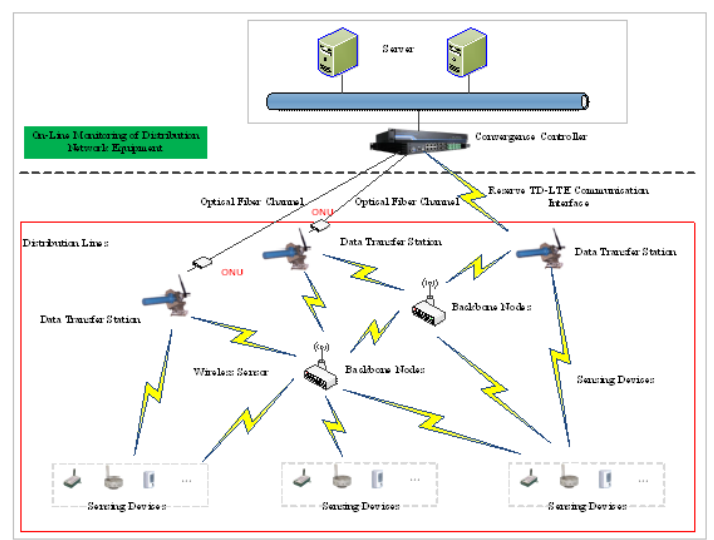

Figure 2 Terminal communication scheme of things

\subsubsection{Functional Design}

On-line monitoring of power distribution equipment includes thermometry of distribution network equipment, tilt monitoring of power distribution tower, temperature and humidity monitoring of distribution network equipment's environment, water immersion state monitoring of distribution network equipment, security monitoring, operating condition monitoring of distribution transformer, and failure condition monitoring of power distribution equipment. Four main aspects to achieve functions are sensor data acquisition, sensor information display, comprehensive analysis of sensor information and alarming information processing.

Condition monitoring of distribution network equipment: installing temperature sensors on temperature-sensitive distribution network equipment to achieve temperature monitoring of operating equipment; installing temperature and humidity sensors on the equipment with higher requirements for operating environment to monitor the operating environment; installing water immersion sensors and noise sensors in RMU, branch box, power distribution room, box change and other facilities to monitor operating state of equipment.

Integrated monitoring of distribution transformer: installing wireless fault current sensors in the side of $10 \mathrm{kV}$ distribution transformer to monitor $10 \mathrm{kV}$ fault current, operating current (anti-electricity stealing data source) and harmonics; installing integrated monitoring nodes in the side of $400 \mathrm{~V}$ to monitor $400 \mathrm{~V}$ working voltage, current, power factor, harmonics and voltage conditions.

Security monitoring: the stealing of RMU, branch boxes and other thefts mainly conducted through breaking into doors and windows, wireless door sensor nodes can be arranged on windows and doors, to alarm theft .

Tower tilt monitoring: tower tilt sensor is installed on tower to achieve the monitoring of tower inclination and it would automatically alarm while the tower inclination exceeds the prewarning value.

\subsection{Life-cycle Management of Distribution Network Equipment}

\subsubsection{Construction Content}

Referring to the State Grid Corporation's code ID specification of relevant material, RFID tag is installed on distribution network equipment to establish equipment identification. In principle, column devices apply active tags, and other devices use passive tags.

Sound ledger information of distribution network equipment should be constructed, device code and asset code should accord with RFID information, to 
provide effective means for the distribution network asset inventory.

Based on RFID identification technology, electronic auxiliary management system covering construction, maintenance, repair, alteration, retire, transfer, scrap and so on should be established, to achieve life-cycle management of distribution network equipment in the demonstration areas.

\subsubsection{Communication Scheme}

RFID device identification information collected by terminal device of field operations uses GPRS wireless public network communication way, through secure access platform to access to the system's master station, to obtain equipment ledger information, monitoring information and operational information; and can promptly return equipment status information and equipment evaluation information collected in the field to the master system.

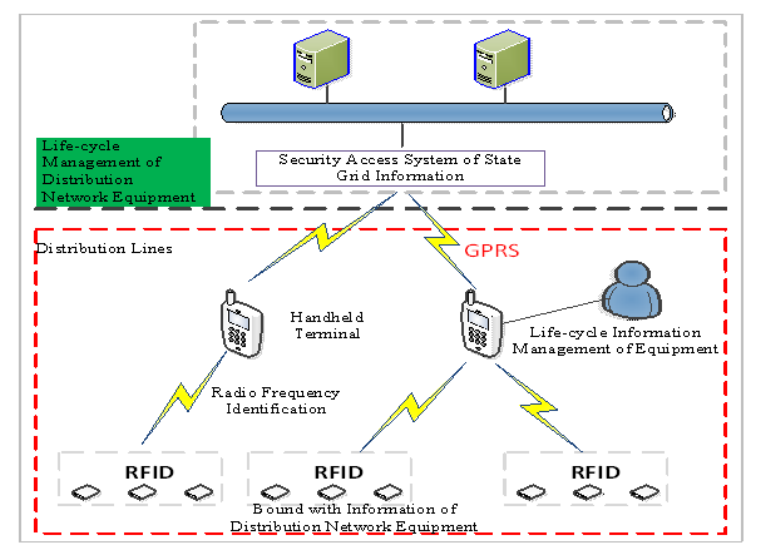

Figure 2 The handheld terminal communication scheme

\subsubsection{Functional Design}

Life-cycle management of distribution network achieves the full life-cycle information maintenance and life-cycle information query of distribution network equipment.

Full life-cycle information maintenance: by collecting operating information at various stages of equipment, and through other business systems, to maintain the full life-cycle information of a device.

Full life-cycle information query: you can query a device's information from procurement to operation until the last scrap, and if the device is in operation, can also check the location information and current operation information of the device.

Terminal of site operations: installing the function of data transfer station into hand-held devices, in order to establish an information channel between signal acquisition devices and monitoring host. It is applied in the aspects of distribution network equipment identification and operating status inquiries.

\section{ACHIEVEMENTS OBTAINED BY IOTIPS DEMONSTRATION PROJECTS}

\subsection{Construction Achievement of Ningxia IOTIPS Demonstration Project}

IOTIPS and the dimensional GIS system, 3D GIS system and unified video platform of Ningxia Electric Power Company have achieved a horizontal integration, covering the Yinchuan East converter station and scene one and two of Yuhuangge distribution lines, realizing the monitoring of device temperature, capacitors deformation, temperature and humidity of environment, water immersion state, line fault current, RMU, cable covers theft and so on, conducting intelligent lock management on maintenance power box inside station, and conducting intelligent management of spare parts.

The implementation of this project can enhance overhaul management and security levels of Yinchuan East converter station, save manpower, material, and optimize resource utilization of spare parts. Meanwhile, it can guide the maintenance work of Yuhuangge line, reduce grid's operation and maintenance costs, reduce outage times, improve power supply reliability rate and support the construction of smart grid.[5]

\subsection{Construction Achievement of Henan IOTIPS Demonstration Project}

Combined with the construction achievement of 2012 IOTIPS demonstration application, Hebi Company is chosen to carry out deepening application of IOTIPS, to solve problems like distribution network equipment operation and management, lack of condition-based maintenance means and so on, enrich information collection tools of urban and rural residents' power utilization, perfect IOTIPS information perception, information transmission and typical application scenarios, and better support the construction of smart grid .

\section{REFERENCES}

[1] Construction of IOTIPS: Integration of Smart Grid and Internet of Things. (2012-07-03) [2012-09-16]. http: // www.chi-nasmartgrid.com.cn/special/?id=370687\& page $=1$.

[2] Revealing the Development Roadmap of Smart Grid of Various Countries (2). (2012-09-14)[2012-09-16]. http://www.chinasmart-grid.com.cn/news/20120914/38832 4-2.shtml.

[3] TANG Yue-zhong, SHAO Zhi-qi, GUO Chuang-xin, et al. Digital Power Grid Architecture. Electric Power Automation Equipment, 2009, 29(6): 115-118.

[4] WANG Jian-zhou. From Internet to IOT http://news.ccidnet.com/art/1032/20090824/1865523.html

[5] SONG He-ying, ZHAO Hui-qun. Program Design and Implementation of Data Acquisition of IOT Distribution Recognizer. Journal of North China University of Technology, 2008, 20 (1): 22-25. 